\title{
Partially Purified Exopolysaccharide from Lactobacillus plantarum YML009 with Total Phenolic Content, Antioxidant and Free Radical Scavenging Efficacy
}

\author{
Byoung-Joo Seo, Vivek Kumar Bajpai, Irfan Ahmad Rather and Yong-Ha Park*
}

Department of Applied Microbiology and Biotechnology, School of Biotechnology, Yeungnam University, Gyeongsan, Gyeongbuk $712-$ 749, KOREA.

\begin{abstract}
This study was aimed to partially purify the exopolysaccharide (EPS) from the culture of Lactobacillus plantarum YML009 using ethanol precipitation method with a yield of $260 \mathrm{mg} / \mathrm{L}$. Analytical evaluationby Bradford and Phenolsulphuric methods revealed the presence of $2.2 \%$ and $68.1 \%$ total protein and total sugar contents in partially purified EPS, respectively. Further, to confirm the biological potential, the EPS was evaluated for its antioxidant activity in various scavenging models including DPPH and nitrite radicals as well as reducing power ability. The partially purified EPS $(5-40 \mathrm{mg} / \mathrm{mL})$ displayed considerable amount of antioxidant efficacy on scavenging DPPH and nitrite radicals by $44.73 \%$, and $43.93 \%$, respectively. Moreover, EPS showed potentreducing power capacity along with total phenolic content $18.96 \mu \mathrm{g} / \mathrm{mg}$ of GAE.
\end{abstract}

Key words: Antioxidant, Exopolysaccharide, Free radical scavenger, Lactobacillus plantarum YMLO09, Total phenolic content.

\section{INTRODUCTION}

A number of exogenous sources in human body produce reactive oxygen species (ROS) with most biological significance and are considered potentially damaging transient chemical species. ${ }^{1}$ A number of chronic human disorders including cancer, cardioand cerebral-vascular diseases have been recognized due to the possible consequence of ROS formation, and/or free radical damage to lipid, protein and nucleic acid. ${ }^{2} \mathrm{~A}$ large number of functional EPS producing LAB have been isolated from various fermented sources which have shown their potent ability to act as antioxidants, probiotics, cholesterol lowering agents, and as a protecting agents against liver damage., ${ }^{3,4}$

Synthesis of exopolysaccharides (EPSs) by lactic acid bacteria (LAB) is well known phenomenon which exists as a cell-bound EPS, adhering closely to the bacterial surface, and released EPS that releases into the surrounding medium. ${ }^{4,5}$ The EPSs are associated with microbial cells protection against the adverse environments including desiccation, toxic materials and osmotic stress. ${ }^{4}$ The EPSs are thought to play a significant role in the colonization of LAB to various ecosystems by facilitating the colonization of LAB to intestinal mucosa, thus enhance the immunity of host. ${ }^{6}$ Now a days the EPSs are used as bio-thickeners due to their stabilizing, emulsifying or gelling properties especially in the food industry. ${ }^{4}$

Some of the EPSs produced by LAB may confer health benefits such as immunemodulatory, anti-tumor, anti-bio film and antioxidant activities. ${ }^{4,7}$ However, antioxidant properties have particularly received huge attention due to the increasing number of diseasesbeing caused by the formation
Submission Date : 05-01-2015 Revision Date : :16-03-2015 Accepted Date : :23-04-2015

DOI: $10.5530 /$ ijper.49.4.6 Correspondence Address Dr. Yong-Ha Park

Department of Applied Microbiology and Biotechnology,

School of Biotechnology, Yeungnam University, Gyeongsan,

Gyeongbuk 712-749,

KOREA.

E-mail:peter@ynu.ac.kr

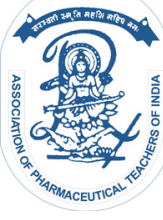

www.ijper.org 


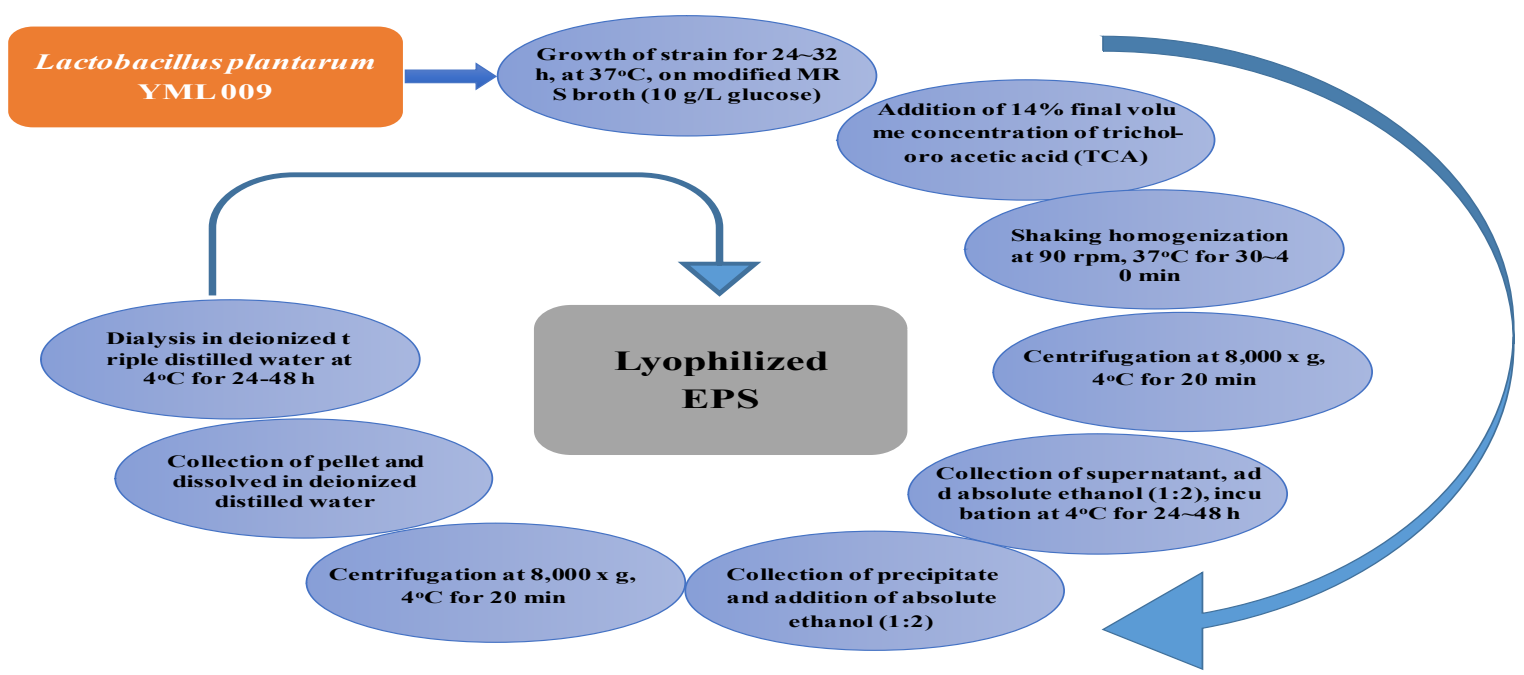

Schematic representation of isolation of partially purified expolysaccharide (EPS) from L. plantarum YMLO09 with antioxidant potential.

\section{Pictorial Abstract}

of free radical and/or reactive oxygen species (ROS). Although synthetic antioxidants have proved effectiveness, adversary side effects have raised severe concerns. Therefore, huge attention has been paid on the use of antioxidants from natural resources such as LAB. ${ }^{7}$ since EPSs from safe natural flora of LAB may serve as potential substitutes to the synthetic antioxidants.

The LAB especially Lactobacillus plantarum YML009 have not been explored to the greater extents for their the rapeutic efficacy, hence, this can be considered first report on the extraction and partial purification of exopolysaccharide (EPS) from Lactobacillus plantarum YML009. The aim of the present study was to isolate partially purified exopolysaccharide (EPS) from a probiotic LAB strain L.plantarum YML009, and to confirm its antioxidant potential in various scavenging models as a natural antioxidant.

\section{MATERIALS AND METHODS}

\section{Microorganism and culture condition}

The bacterial strain Lactobacillus plantarum YML009, previously isolated from Kimchi, a Korean traditional fermented food, and was grown in MRS medium at $37^{\circ} \mathrm{C}$ for $24 \mathrm{~h}$ and maintained on MRS agar medium at $4^{\circ} \mathrm{C}$. The nucleotide sequence was submitted in the Gene Bank with accession number KJ944300.

\section{Reagents and chemicals}

Glucose, trichloroacetic acid (TCA), sodium azide $\left(\mathrm{NaN}_{3}\right)$ bovine serum albumin, 1,1-diphenyl-2-picryl hydrazyl (DPPH), Griess reagent, Folin-Ciocalteu reagent, nitro blue tetrazolium (NBT), ferric chloride, potassium ferricyanide, gallic acid, P-Nitrophenyl- $\alpha-\mathrm{D}$ glucopyranoside, and standard antioxidant compound ascorbic acid were purchased from Sigma-Aldrich, St. Louis, MO, USA. Dialysis membranes ( $\mathrm{Mw}$ cut-off 8000-14,000 Da) were from Spectrum Laboratories Inc. (Rancho Dominguez, CA). All other reagents used were of high analytical grade. Spectrophotometric measurements were done using a 96-well micro-plate enzyme linked immunosorbent assay (ELISA) reader (Infinite M200, Teacan, Mannedorf, Switzerland).

\section{Extraction, isolation and partial purification of exopolysaccharide (EPS)}

Lactobacillus plantarum YML009 was cultured at $37^{\circ} \mathrm{C}$ for $32 \mathrm{~h}$ in MRS modified medium supplemented with $10 \%$ glucose. After centrifugation, the supernatant was collected and added with final concentration of $14 \%$ trichloroacetic acid and left for homogenization in a shaker for 30-40 min followed by centrifugation at 8,000 $\times g$ for 20 min at $4^{\circ} \mathrm{C}$. The supernatant was then added to ethanol (four-fold volume of supernatant) at $4^{\circ} \mathrm{C}$ for $24 \mathrm{~h}$, followed by centrifugation at $8000 \times g$ at $4^{\circ} \mathrm{C}$ for $20 \mathrm{~min}$, resulting in the obtaining of crude precipitate. Finally, the precipitate was dissolved in deionized water and dialyzed using Spectra/Por molecular porous tubular dialysis membrane for $24 \mathrm{~h}$ and lyophilized in a II Shin freeze dryer (Korea). The freeze-dried lyophilized powder of L. plantarum YML009 considered to be partially purified EPS, and was stored at $-80^{\circ} \mathrm{C}$ before use.

\section{Estimation of total protein content}

The total protein content of lyophilized exopolysaccharide (EPS) isolated from L. plantarum YML009 was determined by the protein-dye binding method (Brad- 
ford method) using bovine serum albumin as the standard as described previously. ${ }^{8}$

\section{Estimation of total sugar content}

The total sugar content of lyophilized exopolysaccharide (EPS) isolated from L. plantarum YML009 was determined by phenol-sulfuric acid method using glucose as the standard as described previously. ${ }^{9}$

\section{Antioxidant efficacy}

\section{DPPH radical scavenging assay}

The antioxidant activity of partially purified EPS from L. plantarum YML009, based on the scavenging of stable 2,2-diphenyl-1-picrylhydrazyl (DPPH) free radical, was determined by the method as described previously. ${ }^{1}$ Different concentrations of EPS $(5-40 \mathrm{mg} / \mathrm{mL})$ and reference compound ascorbic acid (25-200 $\mu \mathrm{g} / \mathrm{mL})$ were added to $0.004 \%$ methanolic solution of DPPH (1:1) in a 96-well microplate. The mixture was incubated at $37^{\circ} \mathrm{C}$ in dark for $30 \mathrm{~min}$ with shaking at $150 \mathrm{rpm}$. Absorbance was recorded at $517 \mathrm{~nm}$ using the 96-well ELISA reader against a blank sample. All the tests were run in triplicate. The percent inhibition activity was calculated using the formula.

\section{Nitriteradical scavenging assay}

The nitrite radical scavenging activity of partially purified EPS from L. plantarum YML009 was determined using Griess reagent. ${ }^{10}$ Briefly, $1 \mathrm{~mL}$ of EPS sample at various concentrations $(5,10,20,30$ and $40 \mathrm{mg} / \mathrm{mL}$ ) was mixed with $1 \mathrm{~mL}$ of $1 \mathrm{mM} \mathrm{NaNO}$, solution. Then, $8 \mathrm{~mL}$ of $0.2 \mathrm{M}$ citrate buffer $(\mathrm{pH} 3)$ was added to the mixture, followed by incubation at $37^{\circ} \mathrm{C}$ for $1 \mathrm{~h}$ in a water bath. After incubation, $1 \mathrm{~mL}$ of reaction mixture was added to a mixture of $2 \mathrm{ml}$ of $2 \%(\mathrm{v} / \mathrm{v})$ acetic acid and $0.4 \mathrm{~mL}$ of $1 \%(\mathrm{v} / \mathrm{v})$ Griess reagent. The mixture solution was then vigorously mixed and placed at room temperature for $15 \mathrm{~min}$, after which the absorbance was measured at $520 \mathrm{~nm}$. Ascorbic acid was used as a positive control at the concentration range of 50-500 $\mu \mathrm{g} /$ $\mathrm{mL}$. All the tests were performed in triplicate. The scavenging activity of each sample or positive control was calculated by the following equation:

Scavenging activity $(\%)=\{1-($ Absorbance of treated sample Absorbance of sample or control) / Absorbance of control $\} \times 100$

\section{Reducing power assay}

The reducing power ability of partially purified EPS from L. plantarum YML009 was determined according to the method as described previously with minor modifications. ${ }^{1}$ Briefly, $1 \mathrm{~mL}$ of EPS sample (5, 10, 20, 30 and $40 \mathrm{mg} / \mathrm{mL}$ ) or standard reagent (ascorbic acid as a positive control) at various concentrations $(62.5,125$, 250,500 and $1,000 \mu \mathrm{g} / \mathrm{mL}$ ) was mixed with $2.5 \mathrm{ml}$ of $0.2 \mathrm{M}$ sodium phosphate buffer $(\mathrm{pH}$ 6.6) and $2.5 \mathrm{~mL}$ of $1 \%(\mathrm{w} / \mathrm{v})$ potassium ferricyanide solution. Later, the mixture was incubated at $50^{\circ} \mathrm{C}$ in a water bath for 30 min, mixed with $2.5 \mathrm{~mL}$ of $10 \%$ (w/v) trichloroacetic acid (TCA), and centrifuged at 3,000 xg for $10 \mathrm{~min}$. Then, $250 \mu \mathrm{L}$ of supernatant was mixed with $250 \mu \mathrm{L}$ of DW, after which $500 \mu \mathrm{L}$ of $0.1 \%$ (w/v) $\mathrm{FeCl}_{3}$ was added to the mixture. Ascorbic acid was used as a positive control at the concentration range of $65.5-1000 \mu \mathrm{g} /$ $\mathrm{mL}$. The absorbance was measured at $700 \mathrm{~nm}$. Higher absorbance indicated greater reducing power ability of the samples. All tests were run in triplicate.

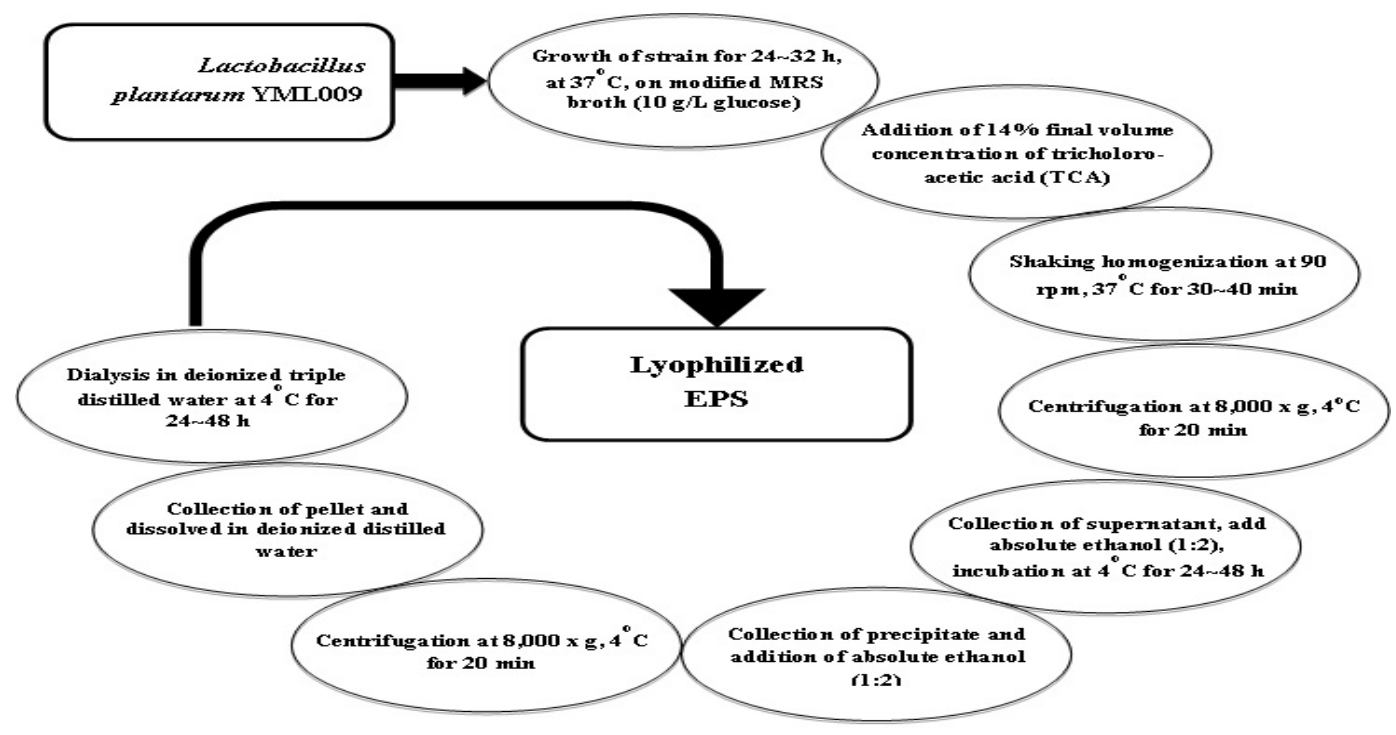

Figure 1: Schematic representation of isolation of partially purified exopolysaccharide (EPS) from L. plantarumYML009 


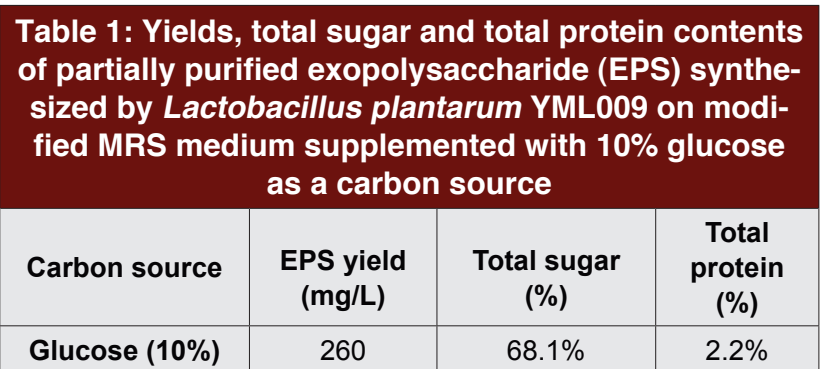

\section{Determination of total phenolic content}

The total phenolic content of partially purified EPS from L. plantarum YML009 was determined using a Folin-Ciocalteu reaction according to previously reported method. ${ }^{1}$ An aliquot $(50 \mu \mathrm{L})$ of EPS (100 $\mu \mathrm{g} / \mathrm{mL}$ ) was mixed with $50 \mu \mathrm{L}$ of $5 \%$ Folin-Ciocalteu reagent and the reaction mixture was incubated $25^{\circ} \mathrm{C}$ for $5 \mathrm{~min}$ in dark followed by addition of $100 \mu \mathrm{L}$ of $20 \%$ $\mathrm{Na}_{2} \mathrm{CO}_{3}$ solution. After incubation at room temperature for $20 \mathrm{~min}$, the absorbance of the developed blue colored chromophore was measured at $730 \mathrm{~nm}$ against an appropriate blank solution. The total phenolic content was evaluated from a standard calibration curve of gallic acid using the concentration range of $50-250 \mu \mathrm{g} / \mathrm{mL}$, and the results were expressed as $\mu$ g gallic acid/mg dry weight sample. All tests were run in triplicate.

\section{Statistical analysis}

All the data were expressed as mean \pm standard deviation of three replicates. Tests of significant differences were determined by one way ANOVA followed by Duncan's test using SAS software (SAS 9.2, SAS Institute Inc., Cary, NC, USA). The values were considered to be significantly different at $\mathrm{p}<0.05$.

\section{RESULTS AND DISCUSSION}

\section{EXTRACTION AND PARTIAL PURIFICATION OF EPS}

It is well known that Lactobacilli are useful microorganisms in dairy technology, along with documented history of use in foods. To date, a number of LAB strains have been reported for their probiotic properties as well as health beneficial effects to human beings. This study was designed to provide important value to the field of probiotics research, with a major objective to extract EPS from L. plantarum YML009, isolated from one of the well-known Korean traditional fermented food Kimchi in order to investigate its health-promoting capability, such as antioxidant effects.

It has also been reported that $\mathrm{LAB}$ produce a great variety of EPSs with different chemical composition and structure, thus providing useful functional properties in food systems.

The present research was designed to isolate and partially purify the EPS from the culture of L. plantarum YML009. Further, the antioxidant activity of this partially purified EPS was evaluated in terms of it's in vitro scavenging abilities on DPPH and nitrite radicals along with its reducing power ability. The most important a primary outcome of the study was that selected culture of LAB (L. plantarum YML009) was efficient producer of EPS than other LAB studied till now. This study led to the isolation of partially purified exopolysaccharide (EPS) from a LAB strain L.plantarum YML009 with a maximum yield of $260 \mathrm{mg} / \mathrm{L}$ on modified MRS medium supplemented with $10 \%$ glucose. Concerning this, YML009 LAB strain could be useful in industrial scale for producing EPS at cheaper cost by using industrial MRS with glucose supplementation. A schematic presentation on the extraction, isolation and partial purification of EPS from L. plantarum YML009 is presented in Figure 1. Although several other LAB have been found to produce EPS in different media composition $^{11}$ the yield of EPS has been dependent on various components of growth medium especially carbohydrate/carbon sources in the medium. The glucose, lactose, and fructose have been considered very frequently used carbon sources with increased EPS yields. ${ }^{11}$ PolakBerecka et al. ${ }^{11}$ found variations in the yields of EPS from L. rbamnosus while using different carbon sources such as galactose, lactose, glucose and maltose with a yield of 81.08, 219.25, 130.08 and $37.37 \mathrm{mg} / \mathrm{L}$, respectively. Lactobacillus casei CG11 in basal minimum medium containing glucose and sucrose at concentrations of $10 \mathrm{~g} / \mathrm{L}$ resulted with EPS yield of 120 and $140 \mathrm{mg} / \mathrm{L}$, respectivelyafter 48 h. ${ }^{12}$ Similarly Lactobacillus rhamnosus $\mathrm{R}$ in basal minimum medium supplemented with glucose or lactose showed about $500 \mathrm{mg} /$ Lof EPS yield. ${ }^{13}$

Moreover, the EPS production by L. fermentum F6 decreased to different levels during the late stationary phase which might be due to the production of glycohydrolases that catalyzed the degradation of polysaccharides, resulting in decreased EPS yield. ${ }^{12,13}$ Degradation of EPS production on prolonged incubation has been reported previously for other LAB strains ${ }^{14,15}$ Since regulation of the EPS biosynthetic pathway in LAB is dependent on the carbohydrate/carbon sources added to the growth medium, ${ }^{11,16,17}$ supplementation of these sources may result in the variations of EPS recovery rate from different $\mathrm{LAB}$ strains.

\section{Total protein content}

In this assay, the partially purified EPS isolated from L.plantarum YML009 showed $2.26 \%$ of total protein 


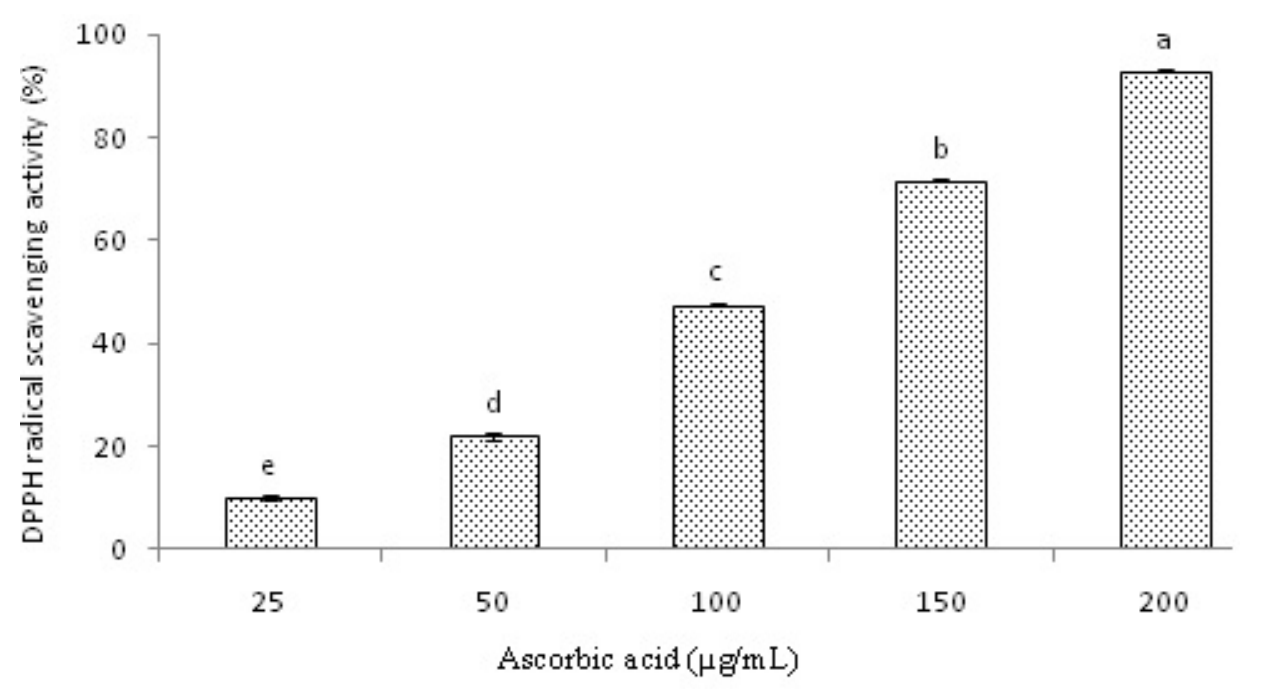

A

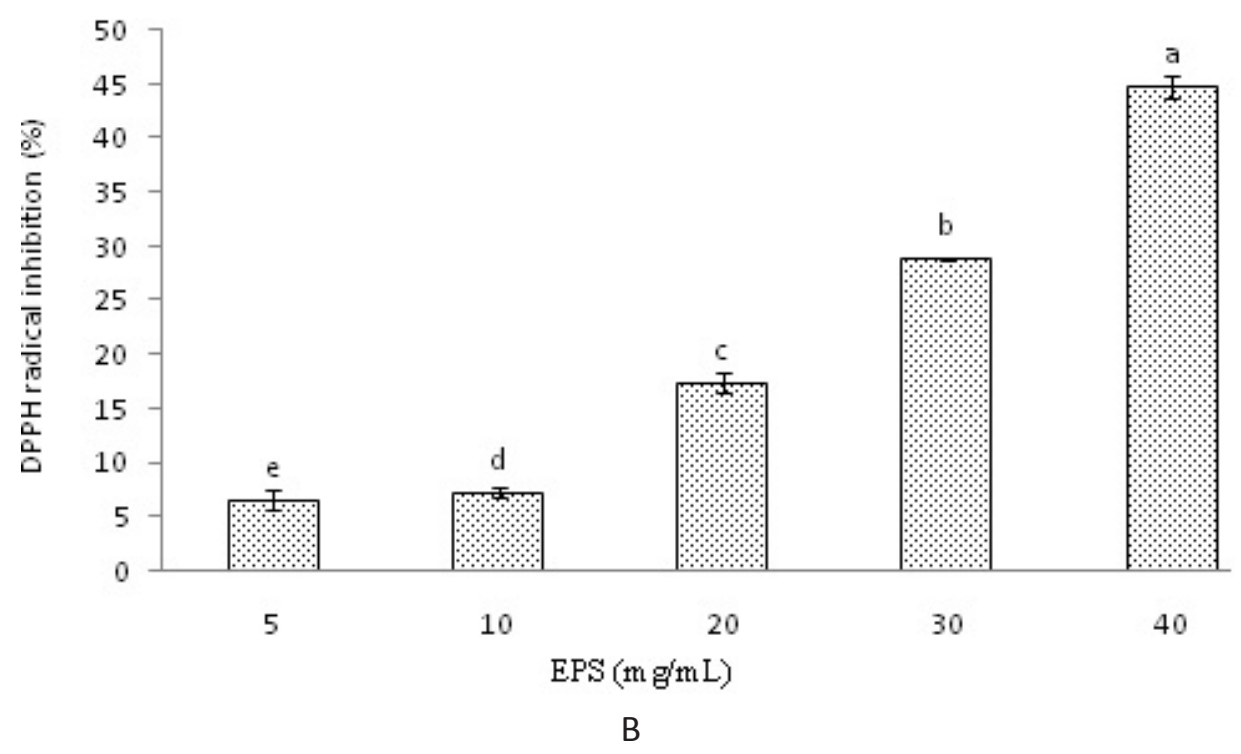

Figure 2: DPPH radical scavenging activity of standard antioxidant compound ascorbic acid (a) and partially purified EPS (b) from L. plantarum YML009

Data are expressed as mean \pm SD $(n=3)$. Values with different superscripts are significantly different $(p<0.05)$.

content when grown on glucose as carbon source in modified MRS growth medium (Table 1). The crude EPS isolated from Lactobacillus helveticus MB2-1 resulted in $3.08 \%$ of total protein content. ${ }^{8}$ Also a lyophilized crude EPS from L. plantarum 301102 S resulted with $1.6 \%$ protein content. ${ }^{18}$ In addition, variations were observed in the EPS protein contents from L. rhamnosus while using galactose, lactose, glucose and maltose as carbon sources and the contents of total protein were found to be $3.88 \%, 2.82 \%, 2.59 \%$ and $6.82 \%$, respectively. ${ }^{11}$ The purity of the EPS is directly proportional to the protein contents present in the EPS. Previously a similar relationship on the protein contents present in the different EPSs isolated from various LAB strains has been reported on the purity of the EPS. ${ }^{11}$

\section{Total sugar content}

In this assay, using phenol-sulfuric method, the EPS isolated from L. plantarum YML009 displayed significant amount of total sugar content. As presented in Table 1, the partially purified EPS from L. plantarum YML009 displayed $68.1 \%$ of total sugar content with lesser protein impurities, which also positively correlate its usefulness for commercial purposes. As reported in previous reports, different LAB strains have been shown to display different amount of total sugar content. ${ }^{8,18}$ EPS derived from $L$. belveticus MB2-1 displayed $71.68 \%$ of total sugar content in crude EPS. ${ }^{8}$ In addition, EPS from simple whey after fermentation by L.plantarum $301102 S$ showed $81 \%$ total sugar content. ${ }^{18}$ Polak-Berecka et al. ${ }^{11}$ Reported variations in total sugar content from L. rhamnosus while using galactose, lactose, glucose and malt- 


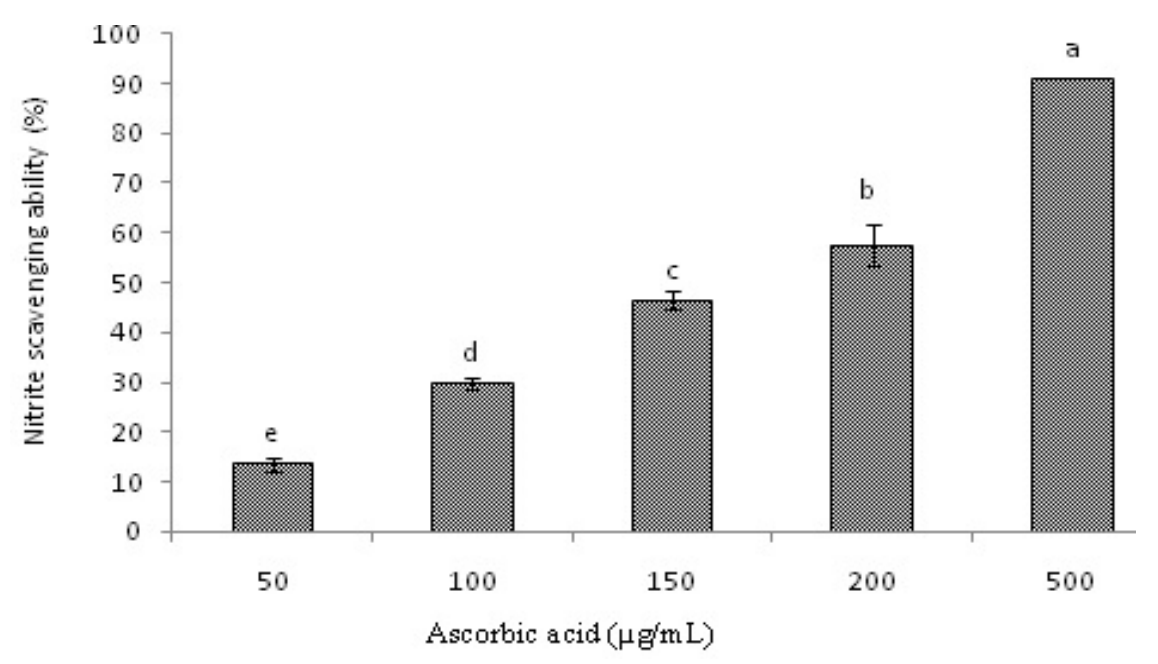

A

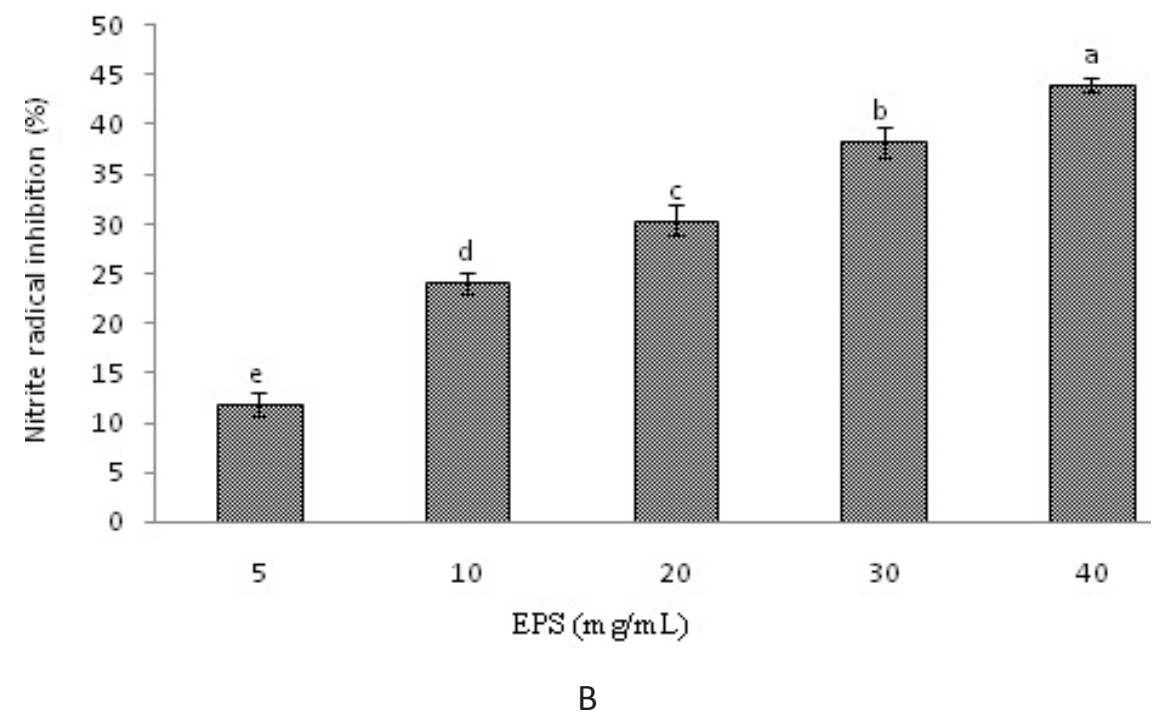

Figure 3: Nitrite radical scavenging activity of standard antioxidant compound ascorbic acid (a) and partially purified EPS (b) from L. plantarum YML009

Data are expressed as mean $\pm S D(n=3)$. Values with different superscripts are significantly different $(p<0.05)$.

ose as carbon sources which were found to be $96.12 \%$, $97.18 \%, 97.41 \%$ and $93.18 \%$, respectively.

\section{Antioxidant potential}

In the natural environment, LAB derived EPS may protect the microbial cell against desiccation, phagocytosis, phage attack, antibiotics or toxic compounds and cellular recognition. ${ }^{19}$ Once we extracted and purified EPS, we need to evaluate its functional and physiological properties by which we can conclude where and in which way it can be used commercially. Here in this research, we analyzed the antioxidant potential of this partially purified EPS in order to characterize its use either as food supplementing agent or in direct drug delivery system. As a result, the partially purified EPS exhibited significantly higher antioxidant capacities in a dose-dependent manner in various in vitro models. These results demonstrated that the EPS from L.plantarum YML009 has antioxidant effects that may involve scavenging of reactive oxygen species (ROS), up-regulation of enzymatic and nonenzymatic antioxidant activities. It has been reported that $\mathrm{LAB}$ and their secondary products not only reduce the risk of ROS accumulation but also degrade superoxide anion and hydrogen peroxide. ${ }^{20}$ Recently huge attention has been focused to evaluate the biological and therapeutic potential of LAB derived EPSs to serve as natural antioxidantsas well as functional food supplements. ${ }^{19,20}$ Polak-Berecka et al. ${ }^{11}$ reported antioxidant effects of EPSs and assumed that the structure-function and composition relationship in EPSs biopolymers play a crucial role for their specific biological actions. Although several studies have shown the antioxidant potential of microbial polysaccharides, ${ }^{21,22}$ very limited information is available concerning the mechanisms of the antioxidant action of polysaccharides at molecular 


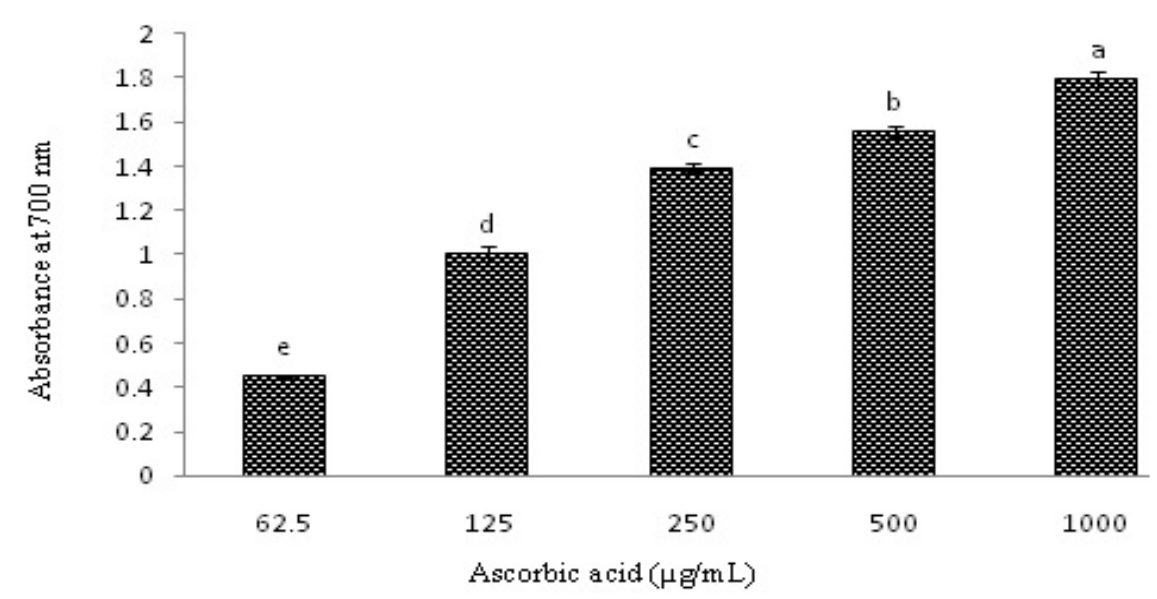

A

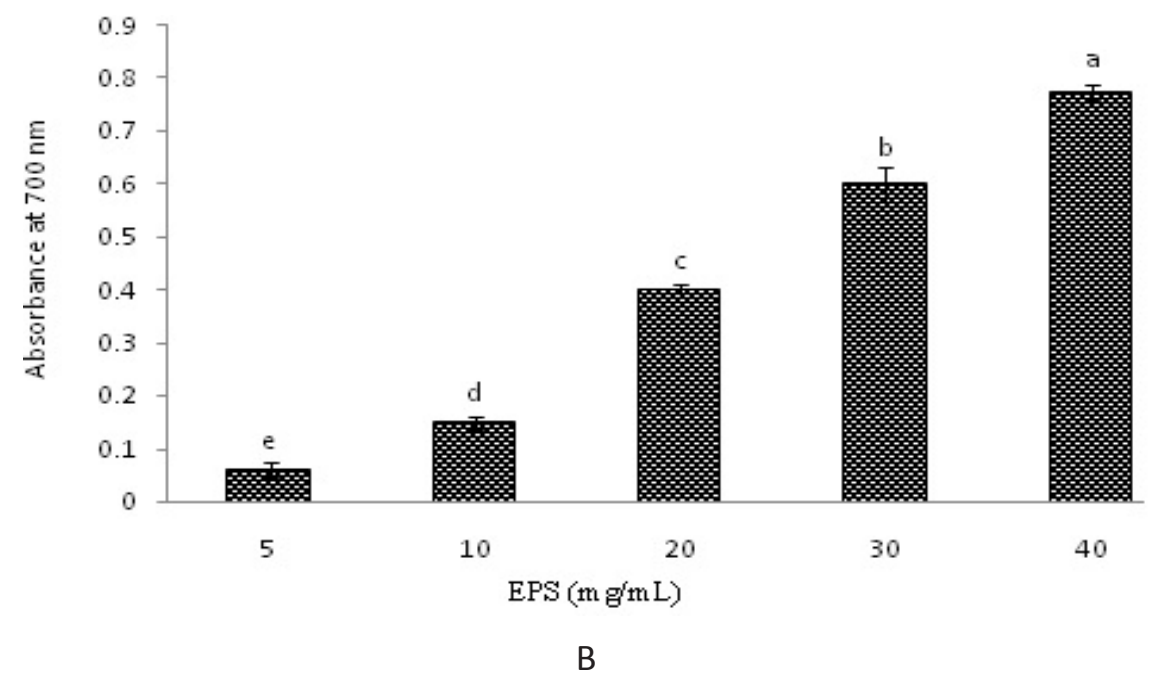

Figure 4: Reducing power ability of standard antioxidant compound ascorbic acid (a) and partially purified EPS (b) from $L$. plantarum YML009

Data are expressed as mean $\pm S D(n=3)$. Values with different superscripts are significantly different $(p<0.05)$

level. In addition, EPS-producing Lactobacillus plantarum C88 isolated from Chinese fermented food was found to display free radical scavenging ability which may involve scavenging of reactive oxygen species (ROS), up-regulation of enzymatic and non-enzymatic antioxidant activities, and inhibition of lipid peroxidation. ${ }^{23}$ Similarly strong and concentration-dependent antioxidant activities of LAB derived EPS have been reported in various antioxidant models in vitro ${ }^{8,24}$ Zhang et al. ${ }^{25}$ also reported the strong antioxidant activity of pure EPS isolated from Lactobacillus plantarum C88. While in the presented research work, we utilized partially purified EPS which was extracted by using industrial MRS supplemented with glucose with lost cost production efficacy and showed efficient antioxidant effect.

Moreover, since the EPS from L. plantarum YML009 showed significant $(<0.05)$ and concentration-dependent antioxidant affects, it can be hypothesized that it can be used in food or medicine industry. In addition, when considering its usefulness in food industry, the partially purified EPS with efficient antioxidant potential will also work well in the form of either food additive, preservative and food supplement. However, due to the low amount of EPS produced by LAB, the use of these substances as food-grade additives is still limited. ${ }^{26}$ Hence, while considering their application in drug delivery or medicine industry, we need to further improve the purity, stability and chemical characterization as to specific applications of these functional EPS could be determined. In addition, limitations have been encountered during EPS purification leading to very less recovery of pure EPS making it difficult for utilizing at industrial scale up. Hence, this is to emphasis that partially purified EPS from L. plantarum YML009 in its present form could be of sufficient use and practical applications at least in food industry but not in drug delivery system.

\section{DPPH radical scavenging activity}

The percentage inhibition of DPPH radical scavenging capacity of partially purified EPS from L. plantarum YML009 in comparison of standard compound ascorbic has been shown in Figure 2. The DPPH free radical scavenging activity of partially purified EPS at 5, 10, 
20,30 and $40 \mathrm{mg} / \mathrm{mL}$ was found to be $6.50 \%, 7.24 \%$, $17.42 \%, 28.84 \%$ and $44.73 \%$, respectively. However, ascorbic acid at 25, 50, 100, 150 and $200 \mu \mathrm{g} / \mathrm{mL}$ displayed $10.03 \%, 22.07 \%, 47.58 \%, 71.64 \%$, and $93.00 \%$ inhibitory effect on scavenging DPPH radical, respectively (Figure 2). In this assay, the EPS showed dosedependent DPPH radical scavenging activity as did by standard compound. Similarly EPS from L. plantarum C88 also showed DPPH free radical scavenging activity in a dose-dependent manner. ${ }^{25} \mathrm{Xu}$ et al. ${ }^{27}$ reported that the DPPH radical scavenging activity of the EPS from Bifidobacterium animalis was as higher as ascorbic acid and was in dose-dependent manner. Li et al. ${ }^{8}$ Also reported dose-dependent DPPH free radical scavenging activity of crude EPS from L. helveticus MB2-1. Significant activity may probably due to the presence of other antioxidant components in the crude EPS which may be proteins, peptides and microelements, there by exhibit potent antioxidant efficacy synergisticallyor by interacting with other compounds present in the crude EPS. ${ }^{8}$

\section{Nitriteradical scavenging activity}

As shown in the Figure 3, both partially purified EPS from L.plantarum YML009 and positive control showed significant nitrite radical scavenging activity in a concentration-dependent manner. In this assay, EPS caused a concentration-dependent inhibitory effect on nitrite radicals. The EPS (5, 10, 20, 30 and $40 \mathrm{mg} / \mathrm{mL}$ ) and ascorbic acid (50, 100, 150, 200 and $500 \mu \mathrm{g} / \mathrm{mL})$ at the tested concentration showed the inhibition of nitrite radicals by $(11.91 \%, 24.08 \%, 30.35 \%, 38.27 \%$ and $43.93 \%)$ and $(13.63 \%, 29.72 \%, 46.57 \%, 57.52 \%$ and 91.19\%), respectively (Figure 3). The radical scavenging activities of EPS-producing LAB have been reviewed previously. ${ }^{28}$

\section{Reducing power activity}

In this assay, EPS from L. plantarum YML009 at 5, 10, 20,30 and $40 \mathrm{mg} / \mathrm{mL}$ showed reducing power ability by 0.06, 0.14, 0.39, 0.59 and 0.77, respectively (Figure 4). On the other hand, the reducing power ability of standard drug ascorbic acid at 62.5, 125, 250, 500 and 1000 $\mu \mathrm{g} / \mathrm{mL}$ was found to be $0.45,1.01,1.39,1.56$ and 1.79 , respectively (Figure 4). These results demonstrated that EPS had marked ferric ions $\left(\mathrm{Fe}^{3+}\right)$ reducing ability along with electron donor properties for neutralizing free radicals by forming stable products. Similarly, Liu and $\operatorname{Pan}^{29}$ also observed the reductive activities of EPS extracted from L. paracasei NTU 101 and L. plantarum NTU 102 using $\mathrm{K}_{3} \mathrm{Fe}(\mathrm{CN})_{6}$ reduction method and demonstrated potential reducing power activity.

In addition, concerning previously reported hypothesis on reducing power ability and facts on functional properties of LAB strains such as L.plantarum NTU 102 also induced superoxide dismutase (SOD) and phenoloxidase (PO) activities as an immune response in Litopenaeus vannamei. Soy-skim milk fermented with $L$. paracasei subsp. paracasei 101 or L. plantarum NTU 102 is useful for the prevention of acute gastric ulcers induced by pylorus ligation significantly enhances SOD based antioxidant activity. ${ }^{30}$ In addition, heat-killed cells and cytoplasmic fractions from these Lactobacillus strains also had inhibitory effects on cancer cell lines and antioxidant activities in vitro. ${ }^{29}$ It has also been analyzed that not only these EPS used in the food industry, but they have also been reported to possess anti-inflammatory, antioxidant, and immunomodulatory activities. ${ }^{31}$ In recent years, the current research in the antioxidant properties of LAB has been hypothesized on the basis that LAB strains and their metabolic products not only reduce the risk of ROS accumulation through food ingestion but also degrade superoxide anion and hydrogen peroxide. Recently Kishk et al..$^{20}$ also focused on antioxidant extracellular polysaccharides with potential applications in the food industry. Here after, based on all the above mentioned prior hypothesis, we confirmed the strong antioxidant potential of low cost partially purified EPS from L. plantarum YML009.

\section{Total phenolic content}

The influence of phenolic content on the antioxidant capacity of LAB-based compounds has been demonstrated previously. Interestingly, the EPS from L. plantarum YML009 showed $18.96 \mu \mathrm{g} / \mathrm{mg}$ of GAE of total phenolic content. Previously it has been confirmed that polyphenolic compounds of LAB have marked antioxidant potential. ${ }^{4}$ The antioxidant capacity of LAB can becorrelated the active phenolic compounds present in crude EPS such as other protein or peptide compounds. Phenolic compounds can donate hydrogen atom to free radicals and thus break the chain reaction of lipid peroxidation and prevent from oxidative deterioration. ${ }^{32}$ High potential of phenolic compounds to scavenge free radicals may be explained by their polyhydroxyl groups. ${ }^{29}$ Moreover, phenolic compounds contribute directly to antioxidative action and inhibit lipid peroxidation. ${ }^{32}$

The knowledge of the relationship between EPS composition and its physical and health-promoting properties can increase the range of biopolymers with desirable functions. The partially purified EPS from L. plantarum YML009 could be used as a strong antioxidant additive or it can be used in antioxidant herbal formulations with enhanced biological and functional properties along with its probiotic effect. Moreover, to increase the novelty and popularity of EPS producing LAB strain, L. plantarum YML009 can be used as a complex starter cultures containing other probiotic strains, 
which may exhibit potent health-promoting characteristics. Since the partially purified EPS from L.plantarum YML009 exhibited strong antioxidant effect thus it can be assumed that it may improve the quality of dairy products such as yogurt and cheese and also can be used as a biofilm protector in dairy industry. Since LAB and their byproducts are considered GRAS, the partially purified EPS from L. plantarum YML009 can be used as directly in its crude powdered form in food cooking as a thickening agent with antioxidant potential for daily health supplement.

The industrial applications of LAB based EPS are limited due to higher production cost and lower recovery processes. Such higher EPS producing strains can be explored to resolve the cost related problem. In future, there is a need for collaborative research with molecular biologists and chemical nature of the isolated and partially purified EPS from L. plantarum YML009 in order to get information regarding the type of EPS isolated, its gene expression, and biological synthesis in LAB cells which may lead to enhanced production of functional EPS with high recovery rate. Although great deal of information are available on genetic biosynthesis of EPSs from LAB, exact biosynthetic mechanism for EPS production in various LAB is not known. However, it is thought that EPS subunits are transported across the membrane by either proton motive forces ${ }^{33}$ or translocated from lactobacillus bacterial membrane embedded lipid carriers by a translocase enzyme. ${ }^{34}$ Biosynthesis of polysaccharides is considered to be energy dependent, requiring one mole of ATP for the conversion of each hexose substrate molecule to hexose phosphate and a further high-energy phosphate bond is needed for the synthesis of each sugar nucleotide. Hence, with the use of molecular techniques such as study of gene expression in important LAB, it might be possible to overcome the down-stream production of biological and functional EPSs. Also elaborative work on partially purified EPS from L. plantarum YML009 in order to achieve better understanding regarding its unique chemical structure, that how these sugar moieties are structurally attached to the proteins of the bacterium may provide innovative information on its industrial use in various industries including food, medical, cosmetics, pharmaceutical, and dairy products and could be as a more acceptable and preferred approach to many additives.

In our research work, we explored to produce cost effective and partially purified EPS from L. plantarum YML009 with significant antioxidant potential suggesting it to be an alternative as a food additives or preservatives. However, in future, the partially purified EPS from L. plantarum YML009 could be of use in various clinical therapies for the treatment of various chronic diseases such as cancer and diabetes as also evident by others. ${ }^{35,36}$ Research can also be elaborated on human trials since most of the LAB based compounds are known as GRAS. Hence, this partially purified may also prove its usefulness in drug discovery sector.

\section{CONCLUSION}

In this study, a partially purified exopolysaccharide (EPS) was isolated from L. plantarum YML009. The basic component analysis of partially purified EPS including total protein and sugar contents conferred it to be a significant producer of good quality of EPS. Moreover, the partially purified EPS demonstrated a considerable amount of phenolic compounds as well as antioxidant efficacy in vitro. Natural antioxidants protect the living system from oxidative stress and associated degenerative diseases therefore LAB-based natural antioxidants may play an important role against oxidative damage caused by free radicals. The antioxidant efficacy of EPS makes it to be a molecule of choice for using in health-care system to serve as potent antioxidantagent. The present research utilized partially purified EPS from L. plantarum YML009 which showed significant antioxidant potential. Although at the used concentrations, the partially purified EPS from L. plantarum YML009, which showed maximum antioxidant potential in the range of $45-70 \%$ in all tested in vitro models, some other reports on purified EPS from other LAS strains showed similar antioxidant effect at lesser concentration. Based on these findings, controversies could be raised on the application of using purified or partially purified EPS which might exert more profound effect, specifically when using it in drug delivery system. However, this has to be confirmed in vivo before raising such hypothesis. Therefore, concerning thesecontroversial evidences, we further need to evaluate the industrial usefulness of both purified and partially purified EPS from L. plantarum YML009. Further in-depth studies will focus on the purification of L. plantarum YML009-drevised EPS in order to confirm its precise mode of action in vivo.

\section{CONFLICT OF INTEREST}

Authors declare that there is no any conflict of interest.

\section{ACKNOWLEDGEMENT}

This research work was supported by the Yeungnam University Post-doctoral Research Grant in 2012. 


\section{SUMMARY}

- A partially purified exopolysaccharide was isolated from a probiotic strain Lactobacillus plantarum YML009.

- The partially purified exopolysaccharide showed $2.2 \% \%$ and $68.1 \%$ total protein and total sugar contents, respectively.

- The partially purified exopolysaccharide exhibited potent antioxidant activities in various scavenging models.

- The partially purified exopolysaccharide also showed $18.96 \mu \mathrm{g} / \mathrm{mg}$ of GAE of phenolic content.

\section{About Authors}

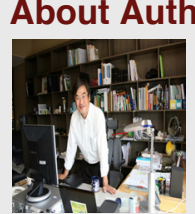

Dr.Yong-Ha Park, is working as a professor in the Department of Applied Microbiology and Biotechnology, Yeungnam University, Republic of Korea. He has published more than 250 peer reviewed research articles in international journals. He has registered more than 30 national and international patents and edited more than 10 academic books. He has been visiting scientist in number of international universities. He is holding the position of VicePresident for the Korean Society for Lactic Acid Bacteria and

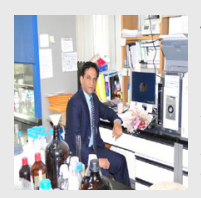

the founder of ProBionic Corporation, Korea. Dr. Vivek K. Bajpai, is working as a Foreign Assistant Professor in the Department of Applied Microbiology and Biotechnology, Yeungnam University, Republic of Korea. He has published more than 100 peer reviewed research/review articles and patent in international journals of scientific repute. $\mathrm{He}$ has been serving as an Associate Editor to one of the world's leading journals BMC Complementary and Alternative Medicine.

\section{REFERENCES}

1. Bajpai VK, Sharma A, Kim SH, Kim Y, Kim JJ, Baek KH. Microwave-assisted seed essential oil of Eleutherococcus senticosus and its antioxidant and free radical scavenging activities. J Food Biochem. 2013; 37(1): 119-27.

2. Sugamura K, Keaney IF. Reactive oxygen species in cardiovascular diseases. Free Rad. Biol. Med. 2011; 51(5): 978-92.

3. Rishi P, Bharrhan S, Singh G, Kaur IP. Effect of Lactobacillus plantarum and L-arginine against endotoxin-induced liver injury in a rat model. Life Sci. 2011; 89(23): 847-53.

4. Wang CL, Huang TH, Liang TW, Fang CY, Wang SL. Production and characterization of exopolysaccharides and antioxidant from Paenibacillus sp. TKU023. 2011; 28(6): 559-65.

5. Tallon R, Bressollier P, Urdaci MC. Isolation and characterization of two exopolysaccharides produced by Lactobacillus plantarum EP56. Res Microbiol. 2003; 154(10): 705-12.

6. Górska S, Jachymek W, Rybka J, Strus M, Heczko PB, Gamian A. Structural and immunochemical studies of neutral exopolysaccharide produced by Lactobacillus johnsonii 142. Carbohydr Res. 2010; 345(1): 108-14.

7. Ramchandran L, Shah NP. Effect of exopolysaccharides and inulin on the proteolytic, angiotensin-I-converting enzyme- and a-glucosidase-inhibitory activities as well as on textural and rheological properties of low-fat yogurt during refrigerated storage. Dairy Sci Technol. 2009; 89(6): 583-600.

8. Li W, Ji J, Chen X, Jiang M, Rui X, Dong M. Structural elucidation and antioxidant activities of exopolysaccharides from Lactobacillus helveticus MB2-1. Carbohydr Polym. 2014; 15(2): 351-9.

9. Xu RH, Shen Q, Ding XL, Gao WG, Li PL. Chemical characterization and antioxidant activity of an exopolysaccharide fraction isolated from Bifidobacterium animalis RH. Eur Food Res Technol. 2011; 232(10): 231-40.

10. Choi D, Cho KA, Na MS, Choi HS, Kim YO, Lim DH, et al. Effect of bamboo oil on anti-oxidative activity and nitrite scavenging activity. J Ind Eng Chem. 2008; 14(6): 765-70.

11. Polak-Berecka M, Wasko A, Szwajgier D, Choma A. Bifidogenic and antioxidant activity of exopolysaccharides produced by Lactobacillus rhamnosus E/N cultivated on different carbon sources. Polish J Microbiol. 2013; 62(2): 181-9.

12. Cerning J, Renard CMGC, Thibault JF, Bouillanne C, Landon M, Desmazeaud M, Topisirovic L. Carbon source requirements for exopolysasccharide production by Lactobacillus case $i$ CG11 and partial structure analysis of the polymer. Appl Environ Microbiol. 1994; 60(2): 3914-9.

13. Pham PL, Dupont I, Roy D, Lapointe G, Cerning J. Exopolysaccharide by Lactobacillus rhamnosus $\mathrm{R}$ and analysis of its enzymatic degradation during prolonged fermentation. Appl Environ Microbiol. 2000; 66(6): 2302-10.

14. Mozzi F, Rollán G, Savoy de Giori G, Font de Valdez G. Effect of galactose and glucose on the exopolysaccharide production and the activities of

biosynthetic enzymes in Lactobacillus case i CRL 87. J Appl Microbiol. 2001; 91(1): 160-7.

15. Degeest B, Janssens B,De Vuyst L. Exopolysaccharide (EPS) biosynthesis by Lactobacillus sakei 0-1: production kinetics, enzyme activities and EPS yields. J Appl Microbiol. 2001; 91(3): 470-7.

16. Grobben GJ, Sikkema J, Smith MR, De Bont JAM. Production of extracellular polysaccharides by Lactobacillus delbrueckii ssp. bulgaricus NCFB 2772 grown in a chemically defined medium. J Appl Bacteriol. 1995; 79(1): 103-7.

17. Welman AD, Maddox IS. Exopolysaccharides from lactic acid bacteria: perspectives and challenges. Trends Biotechnol. 2003; 2(6): 269-74.

18. Tsuda H, Miyamoto T. Production of exopolysaccharide by Lactobacillus plantarum and the prebiotic activity of the exopolysaccharide. Food Sci Technol Res. 2010; 16(8): 87-92.

19. Liu CF, Tseng KC, Chiang SS, LeeBH, Hsua WH, Pana TM. Immunomodulatory and antioxidant potential of Lactobacillus exopolysaccharides. J Sci Food Agric. 2011; 91(12): 2284-91

20. Kishk YFM, Al-Sayed HMA. Free-radical scavenging and antioxidative activities of some polysaccharides in emulsions. LWT Food Sci Technol. 2007; 40(2): 270-7.

21. Guo S, Mao W, Han Y, Zhang X. Structural characteristics and antioxidant activities of the exracellular polysaccharides produced by marine bacterium Edwardisiellatarda. Biores Technol. 2010; 101(12): 4729-32.

22. Pan D, Mei X. Antioxidant activity of an exopolysaccharide purified from Lactococcus lactis subsp. lactis 12. Carbo hydr Polym. 2010; 80(3): 908-14.

23. Li S, Zhao Y, Zhang L, Zhang X, Huang L, Li D, et al. Antioxidant activity of Lactobacillus plantarum strains isolated from traditional Chinese fermented foods. Food Chem. 2012; 135(3): 1914-9.

24. Tsai CC, Chan CF, Huang WY, Lin JS, Chan P, Liu HY, et al. Applications of Lactobacillus rhamnosus spent culturesupernatant in cosmetic antioxidation, whitening andmoisture retention applications. Molecules 2013; 18(11): 14161-71.

25. Zhang L, Liu C, Li D, Zhao Y, Zhang X, Zeng X, Yang Z, Li S. Antioxidant activity of an exopolysaccharide isolated from Lactobacillus plantarum C88. Int J Biol Macromol. 2013; 54(1): 270-5.

26. Ruas-Madiedo P, Hugenholtz J, Zoon P. An overview of the functionality of exopolysaccharides produced by lactic acid bacteria. Int Dairy J. 2002; 12(2): 163-71.

27. Xu L, Wang Q, Xiao J, Liu Q, Wang X, Chen T, Zhang Y. Charcterization of Edwardsielle tarda waaL: roles in lipopolysaccharide biosynthesis, stress adaptation, and virulence toward fish Arch Microbiol. 2010; 192(12): 1039-47.

28. Rodriguez H, Curiel JA, Landete JM, de las Rivas B, de Felipe FL, GomezCordoves $\mathrm{C}$, et al. Food phenolics and lactic acid bacteria. Int $\mathrm{J}$ Food Microbiol. 2009; 132(2): 79-90. 
29. Liu CF, Pan TM. In vitro effects of lactic acid bacteria on cancer cell viability and antioxidant activity. J Food Drug Anal. 2010; 18(2): 77-86.

30. Liu CF, Hu CL, Chiang SS, Tseng KC, Yu RC, Pan TM. Beneficial preventive effects of gastric mucosal lesion for soy-skim milk fermented by lactic acid bacteria. J Agric Food Chem. 2009; 57(10): 4433-8.

31. Chabot S, Yu HL, de Leseleuc L, Cloutier D, Van Calsteren MR, Lessard M. Exopolysaccharides from Lactobacillus rhamnosus RW-9595M stimulate TNF, IL-6 and IL-12 in human and mouse cultured immune competent cells, and IFN-c in mouse splenocytes. Lait 2001; 81(12): 683-7.

32. Wojdylo A, Oszmianski J, Czemerys R. Antioxidant activity and phenolic compounds in 32 selected herbs. Food Chem. 2007; 105(3): 940-49.
33. McGrath BC, Osborn MJ. Evidence for energy-dependent transposition of core lipopolysaccharide across the inner membrane of Salmonella typhimurium. J Bacteriol 1991; 173(10): 3134-7.

34. Liu D, Cole RA, Reeves PR. An O-antigen processing function for Wzx (RfbX): a promising candidate for O-unit flippase. J Bacteriol. 1996; 178(7): 2102-107.

35. Ahmadi MA, Ebrahimi MT, Mehrabian S, Tafvizi F, Bahrami H, Dameshghian M. Antimutagenic and anticancer effects of lactic acid bacteria isolated from Tarhana through Ames test and phylogenetic analysis by 16S rDNA. Nutr Cancer. 2014; 66(8): 1406-13.

36. Honda K, Moto M. Uchida N, He F, Hashizume N. Anti-diabetic effects of lactic acid bacteria in normal and type 2 diabetic mice. J Clin Biochem Nutr. 2012; 51(2): 96-101. 